\title{
Hex is a transcriptional repressor that contributes to anterior identity and
}

\section{suppresses Spemann organiser function}

\author{
Joshua M. Brickman', C. Michael Jones ${ }^{2,3}$, Melanie Clements ${ }^{1}$, J. C. Smith² and Rosa S. P. Beddington ${ }^{1, *}$ \\ ${ }^{1}$ Divisions of Mammalian Development and ${ }^{2}$ Developmental Biology, National Institute for Medical Research, The Ridgeway, \\ Mill Hill, London NW7 1AA, UK \\ ${ }^{3}$ Section of Gene Function and Regulation, Institute of Cancer Research, Chester Beatty Laboratories, 237 Fulham Road, London \\ SW3 6JB, UK \\ *Author for correspondence (e-mail: r-beddin@nimr.mrc.ac.uk)
}

Accepted 10 March 2000; published on WWW 10 May 2000

\section{SUMMARY}

One of the earliest markers of anterior asymmetry in vertebrate embryos is the transcription factor Hex. We find that Hex is a transcriptional repressor that can be converted to an activator by fusing full length Hex to two copies of the minimal transcriptional activation domain of VP16 together with the flexible hinge region of the $\lambda$ repressor (Hex- $\lambda$ VP2). Retention of the entire Hex open reading frame allows one to examine Hex function without disrupting potential protein-protein interactions. Expression of Hex- $\lambda \mathrm{VP} 2$ in Xenopus inhibits expression of the anterior marker Cerberus and results in anterior truncations. Such embryos have multiple notochords and disorganised muscle tissue. These effects can occur in a cell non-autonomous manner, suggesting that one role of wildtype Hex is to specify anterior structures by suppressing signals that promote dorsal mesoderm formation. In support of this idea, over-expression of wild-type Hex causes cell non-autonomous dorso-anteriorisation, as well as cell autonomous suppression of dorsal mesoderm. Suppression of dorsal mesoderm by Hex is accompanied by the down-regulation of Goosecoid and Chordin, while induction of dorsal mesoderm by Hex- $\lambda$ VP2 results in activation of these genes. Transient transfection experiments in ES cells suggest that Goosecoid is a direct target of Hex. Together, our results support a model in which Hex suppresses organiser activity and defines anterior identity.

Key words: Anterior pattern, Transcriptional repression, Homeobox, Endoderm, Organiser, Xenopus laevis

\section{INTRODUCTION}

Traditional models for anterior patterning in the vertebrate embryo view the anterior-posterior axis arising as a consequence of gastrulation. Anterior identity was thought to be determined by the position of involuting tissues relative to the embryonic signaling centre, or organiser (Beddington and Smith, 1993). The organiser, initially defined in Amphibia, comprises a group of cells capable of inducing a complete secondary embryonic axis in heterotopic grafting experiments (Spemann and Mangold, 1924). The ability of this cell population to induce a complete secondary axis suggests that the organiser contains all signals necessary to pattern the anterior-posterior axis. However, recent evidence, derived principally from the mouse, suggests that the classically defined organiser (or node in the mouse) is insufficient to pattern the anterior of the embryo. Instead, the signals required for the initiation of anterior patterning are localised to an extraembryonic tissue, the anterior visceral endoderm (AVE), a full day prior to the initiation of overt gastrulation and the formation of the organiser (reviewed by Beddington and Robertson 1998, 1999). Comparative expression analysis of a number of gene products implicated in anterior identity suggest that other vertebrates have structures that may be functionally analogous to the AVE: the anterior hypoblast in chick (Yatskievych et al., 1999), the yolk syncytial layer in zebrafish (Yamanaka et al., 1998), and a deep endodermal population in the frog (Bouwmeester et al., 1996; Jones et al., 1999). The visceral endoderm of the rabbit (Knoetgen et al., 1999), mouse (Tam and Steiner, 1999) and the deep endoderm of the frog (Jones et al., 1999) have been shown to have anterior signaling capabilities.

One of the earliest markers of anterior/posterior asymmetry in a number of vertebrates is the homeobox gene Hex (Thomas et al., 1998). In the mouse, Hex RNA is initially expressed throughout the primitive endoderm at $4.5 \mathrm{dpc}$ and later becomes restricted to a population of visceral endoderm cells at the distal tip, more than a day before the onset of gastrulation. These cells then undergo a directional migration before coming to rest on the presumptive anterior side of the embryo. This displacement precedes expression of Brachyury, a marker of the primitive streak, and the initiation of gastrulation, by at least half a day. In Xenopus a similar movement of an endodermal population expressing XHex is observed (Jones et al., 1999).

Hex is a homeodomain protein of the Antennapedia/Ftz class 
(Crompton et al., 1992; Neidle and Goodwin, 1994). In both mouse and frog, Hex expression resembles that of Cerberus, a gene previously linked to anterior patterning (Belo et al., 1997; Biben et al., 1998; Bouwmeester et al., 1996; Jones et al., 1999; Pearce et al., 1999; Shawlot et al., 1998; Thomas et al., 1997; Zorn et al., 1999) and indeed in Xenopus, ectopic expression of XHex induces Cerberus (Jones et al., 1999; Zorn et al., 1999). In addition to its early endodermal expression in a number of vertebrates, Hex, like Cerberus, is expressed in the earliest anterior-most migrating mesendoderm (Jones et al., 1999; Thomas et al., 1998; Zorn et al., 1999). Hex expression subsequently continues in the gut, liver and thyroid primordia (Newman et al., 1997; Thomas et al., 1998).

Here we use a combination of in vitro cell culture experiments and over-expression in Xenopus to explore further the role of Hex in anterior patterning. We show that Hex is a transcriptional repressor with likely targets that include genes that respond to classical organiser inducing signals. By employing fusion proteins containing the Hex coding sequence and reiterated modular units of the transcriptional activation domain of VP16 (Hex-VP16), we demonstrate a correlation between ectopic expression of potential Hex target genes, a loss of Cerberus expression in deep endoderm, and anterior truncations. Anterior truncations induced by Hex-VP16 contain expanded dorsal mesoderm structures, whereas embryos injected with RNA encoding Hex are anteriorised and display a disruption of dorsal mesoderm at the site of injection.

Injection of RNAs encoding Hex or Hex-VP16 derivatives has allowed us to identify two potential targets of Hexmediated transcriptional repression: Goosecoid (Gsc) and Chordin (Chd). Transient transfection in ES cells using the Gsc promoter suggests that the effects of Hex on Gsc are direct. Expression analyses show that Hex and Gsc are expressed in separate populations during gastrulation, and we suggest that the two genes mark distinct regions required for anterior patterning. Together, our results support a model in which Gsc and Chd mark a default state of trunk organiser, with Hex specifying anterior structures by suppressing the expression of these genes in anterior endoderm.

\section{MATERIALS AND METHODS}

\section{Plasmid construction}

All fusion proteins were constructed with PCR fragments generated to have an EcoRI site upstream of a perfect Kozak sequence, followed by an ATG and a second codon encoding Val. This was followed by the mHex sequence. These were cloned as EcoRI/BamHI fragments into either pBGX-1, to make GAL4 fusions, or into the pBGX-1 based GAL4-VP derivatives (Emami and Carey, 1992). The Hex- $\lambda$ VP fusions were then removed from GAL4 and inserted into pCS2 (Rupp et al., 1994) as an EcoRI/XbaI fragment.

Reporter constructs were designed using pGL-3 (Promega). GAL4 sites were excised from the GxE4 series of reporters described previously (Carey et al., 1990). The Gsc promoter deletions were constructed by removing the promoter sequences (SV40) from pGL3 and inserting upstream sequences from the Gsc promoter (Watabe et al., 1995), including the TATA box, and transcription start site. All deletion derivatives were synthesized in the identical context to the full length promoter using a PCR based strategy.

Point mutations were constructed using a PCR based mutagenesis strategy in which overlapping oligonucleotides containing the point mutation were used to generate a PCR product in which the desired bases had been modified.

Capped synthetic RNA was prepared according to Smith (1993). CS2 Hex derivatives were linearized with NotI and RNA encoding nuc $\beta$-gal was transcribed from pSP6nuc $\beta$ GAL (Smith and Harland, 1991).

\section{Embryonic manipulations}

Xenopus laevis embryos were obtained by in vitro fertilization (Smith and Slack, 1983). Embryos were cultured in 10\% normal amphibian medium (NAM; Slack et al., 1984) and staged according to Nieuwkoop and Faber (1994). Embryos were injected at the 2-, 4-, 8-, 16- and 32-cell stages with $10 \mathrm{nl}$ of RNA in water. RNA concentrations ranged from $10 \mathrm{pg} / \mathrm{blastomere}$ to $500 \mathrm{pg} / \mathrm{blastomere}$ and microinjections were performed according to Smith (1993). Nuclear $\beta$-galactosidase RNA was injected at concentrations of 100 pg/blastomere. For animal cap assays, embryos were dissected and cultured in $75 \%$ NAM or $75 \%$ NAM containing BSA when activin was included in the culture medium. A crude preparation of activin A was made from conditioned medium of COS cells transfected with a human inhibin $\beta_{\mathrm{A}}$ cDNA. Activin units are defined by Cooke et al. (1987).

\section{RNA preparations and RT-PCR}

RNA was extracted from animal caps by the acid guanidinium thiocyanate-phenol-chloroform method (Chomczynski and Sacchi, 1987). Primers for RT-PCR were designed with Primer 3 (Whitehead Web Page); sequences are available upon request. RT-PCR was performed with Titan RT-PCR enzyme mix (Boehringer Mannheim) according to the manufacturer's instructions. Gsc PCR was done for 27 cycles with an annealing temperature of $58^{\circ} \mathrm{C} . E F-1 \alpha$ was carried out with the same number of cycles and an annealing temperature of $55^{\circ} \mathrm{C}$. Primers for $E F-1 \alpha$ were described previously (Jones et al., 1999).

\section{In situ hybridization and lineage tracing}

Whole-mount in situ hybridization was performed essentially as described by Harland (1991). Cerberus (Bouwmeester et al., 1996), Gsc (Cho et al., 1991), Chd (Sasai et al., 1994), Noggin (Smith et al., 1993) and BMP4 (Jones et al., 1996) probes were as described. Fluorsecein-lysine-dextran was used as a lineage tracer and was revealed using anti-fluorescein antibody (Jones and Smith, 1998). In situ hubridisation was carried out on $10 \mu \mathrm{m}$ paraffin sections as described by Jones et al. (1999).

\section{Histology, $\beta$-galactosidase staining and immunohistochemistry}

For histological analysis, specimens were fixed, sectioned and stained as described by Smith (1993). Staining for $\beta$-galactosidase was carried out as described by Beddington et al. (1989). Embryos were fixed in $1 \%$ paraformaldehyde, $0.2 \%$ glutaraldehyde, $2 \mathrm{mM} \mathrm{MgCl}_{2}, 5 \mathrm{mM}$ EGTA and 0.02\% NP-40 (Tada et al., 1997) and washed in PBS with $0.02 \%$ NP-40. Staining was done overnight at room temperature. Whole-mount immunohistochemistry with the monoclonal antibody MZ15 (Smith and Watt, 1985) was performed as described by Smith (1993).

\section{Transfections and cell culture}

Feeder-independent ES cells were maintained on gelatinised flasks in Dulbecco's modified Eagle's medium (DMEM) supplemented with $20 \%$ fetal calf serum and leukemia inhibiting factor (Smith et al., 1988). ES cell lines were E14.2 (Fisher et al., 1989) and CGR8 (Mountford et al., 1994). Transient transfections were done by lipofection using Lipofectamine (Gibco BRL) essentially according to the directions of the manufacturer. Cells were seeded at a density of $1.5 \times 10^{5} / \mathrm{ml}$ and allowed to grow for approximately 24 hours prior to application of the transfection cocktail. Transfections were done 
overnight and the following morning the transfection cocktail was replaced with fresh medium. Transfections contained $100 \mathrm{ng}$ of reporter and $75 \mathrm{ng}$ of internal reference plasmid, with a total of 1.2 $\mu \mathrm{g}$ DNA (see Brickman et al., 1999). Luciferase assays were conducted with Dual Luciferase reagent (Promega) according to the directions of the manufacturer. An internal reference plasmid consisting of the SV40 promoter driving Renilla luciferase (Promega) was used as an internal control for transfection efficiency. To control for variations in transfection efficiency all data are represented as relative activities. Units of relative activity are calculated by dividing firefly luciferase readings by the reference Renilla activities and multiplying by a constant. Fold induction was determined by normalizing the baselines of each experiment to unity. All transfections were carried out in duplicate.

\section{RESULTS}

\section{Design and in vitro function of Hex fusions to reiterated modular activation domains}

To test its ability to regulate transcription in an embryonic cell type, Hex was fused to the heterologous DNA binding domain of GAL4 (Ptashne, 1992) and the resulting GAL4-Hex fusion proteins were assayed using GAL4 site-containing reporters in ES cell culture. ES cells were chosen as a substrate cell line because they express Hex and because they represent an in vitro system that resembles conditions in the early embryo. Fig. 1A shows transcriptional repression by GAL4-Hex. GAL4-Hex and a reporter gene containing five consensus GAL4 sites upstream of the $S V 40$ promoter driving luciferase were transfected into ES cells. Transfection of increasing amounts GAL4-Hex led to repression of the $S V 40$ promoter by up to fivefold. These results extend the findings of Tanaka et al. (1999) who demonstrated that rat Hex is a repressor in HepG2 cells.

To convert Hex to a transcriptional activator without disrupting Hex protein structure we employed the strategy shown in Fig. 1B. Hex was fused to the flexible linker domain from $\lambda$ repressor and the minimal activation domain from VP16. The linker domain gives $\lambda$ the flexibility to bind cooperatively to non-adjacent DNA sites (Astromoff and Ptashne, 1995), and in the context of the VP16 fusions it enhances the ability of an activation domain to stimulate transcription without its own intrinsic activation function (Emami and Carey, 1992; Ohashi et al., 1994). Reiteration of a functional unit containing the $\lambda$ repressor linker and minimal VP16 activation domain has been used previously to analyse the synergy between multiple activation domains bound to a single DNA binding domain (Emami and Carey, 1992; Ohashi et al., 1994). In principle these activation domain modules should allow the recapitulation of an enhancer bound to a single DNA site by allowing the simultaneous interaction of multiple activation domains with the transcription complex (see Fig. 1B). Hex-VP16 fusion proteins were constructed either with or without additional fusion to the DNA binding domain of GAL4 and with either two or four (Hex- $\lambda$ VP2 and Hex- $\lambda$ VP4) activation domain modules. The tripartite GAL4Hex- $\lambda$ VP fusions were constructed to monitor DNA binding from the well-defined high affinity GAL4 site, in addition to native Hex binding sites.

DNA encoding either GAL4, GAL4-Hex- $\lambda$ VP2 or GAL4Hex $-\lambda$ VP4 fusion proteins was co-transfected alongside the indicated reporters. Co-transfection of both GAL4-Hex- $\lambda$ VP2
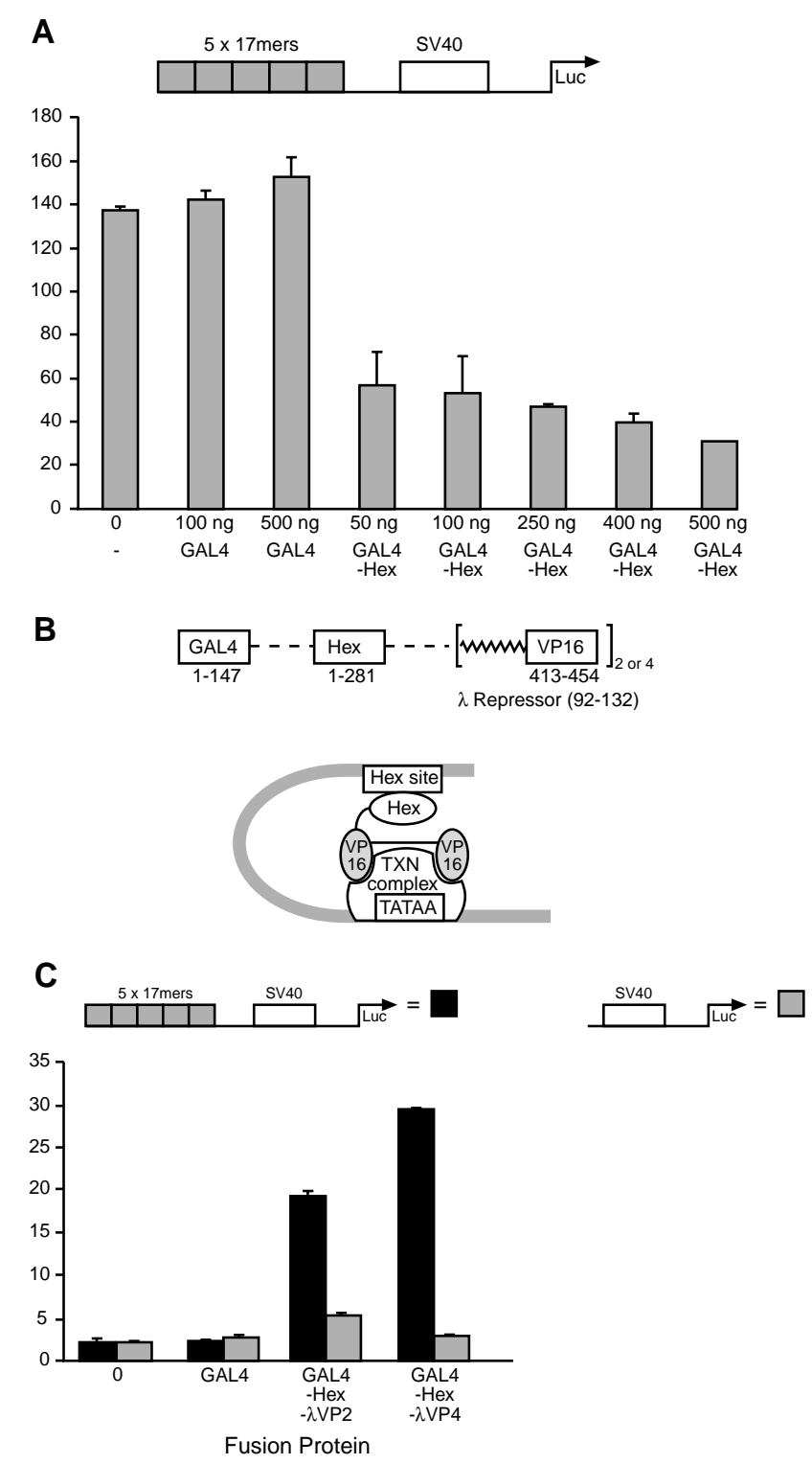

Fig. 1. Hex acts as a transcriptional repressor in ES cells. (A) GAL4Hex represses transcription from upstream of the $S V 40$ promoter. Increasing concentrations of DNA expressing either GAL4 or GAL4Hex were co-transfected with the depicted reporter. (B) Cartoon illustrating the construction and principle behind the design of Hex$\lambda$ VP fusions. (C) Activity of GAL4, GAL4-Hex $\lambda$ VP2 and GALHex $\lambda$ VP4 in ES cells. DNA encoding the indicated fusion protein was co-transfected with the indicated reporters as in A. Bars represent mean activities in arbitrary units (see Materials and Methods); standard deviations are indicated. Amount of plasmids expressing the indicated GAL4 or Hex derivatives are indicated in A and is $100 \mathrm{ng}$ for all transfections in $\mathrm{C}$.

and GAL4-Hex- $\lambda$ VP4 led to approximately tenfold GAL4 site dependent induction of the $S V 40$ promoter (Fig. 1C). There is also a small effect of these molecules on the $S V 40$ promoter due to the presence of a DNA site for Hex within SV4O (data not shown). Both these fusion proteins, and versions in which GAL4 has been removed, are active on native Hex binding sites (see Fig. 7).

Full length VP16 is one of the most potent transcriptional 


\section{Dorsal Hex- $\lambda$ VP2 injection}
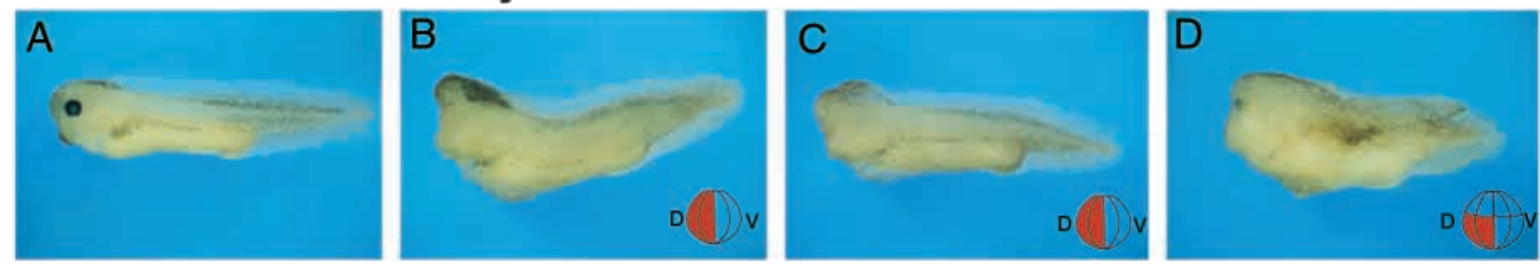

\section{Hex- $\lambda$ VP2 + $\beta$-galactosidase}
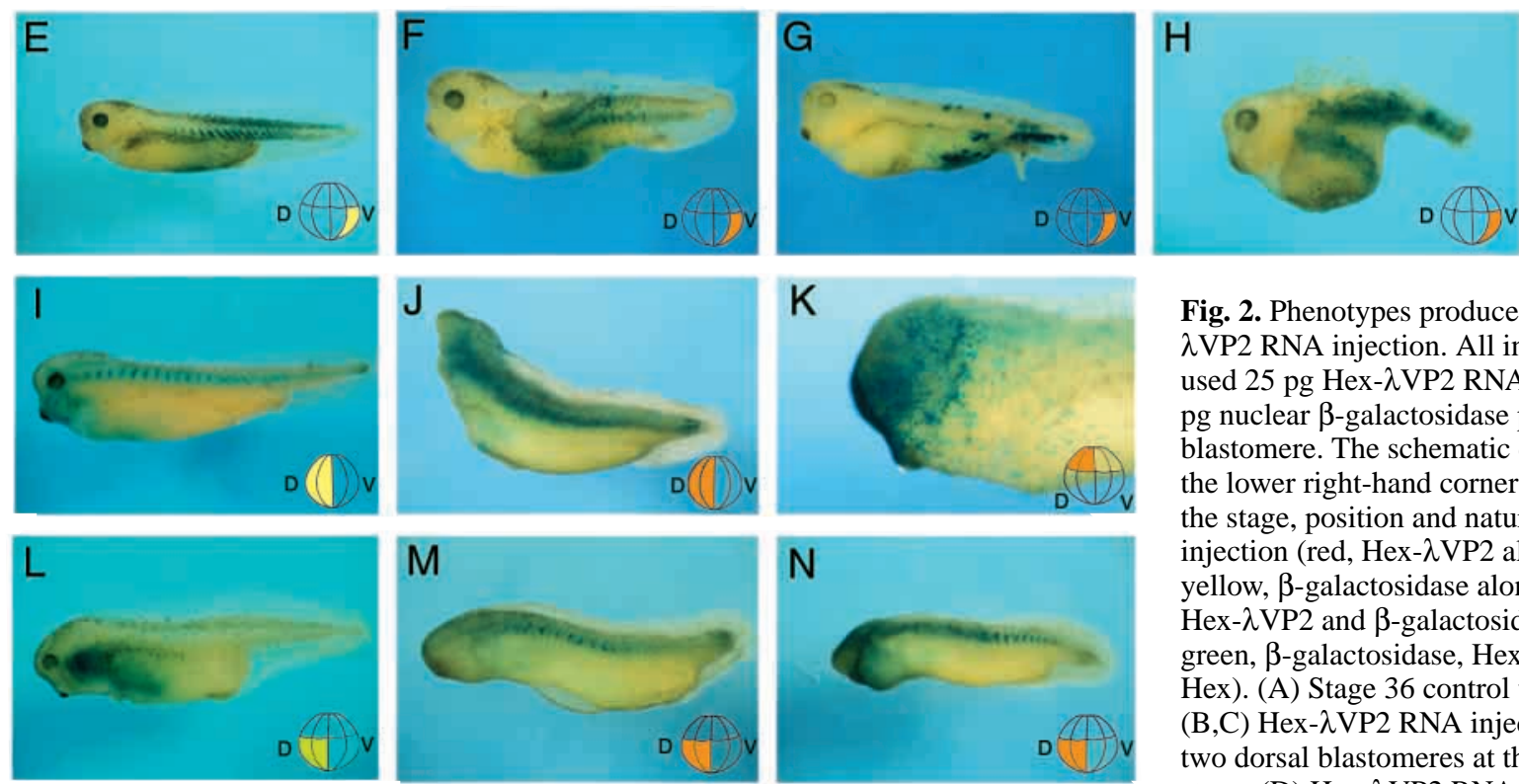

Fig. 2. Phenotypes produced by Hex$\lambda$ VP2 RNA injection. All injections used $25 \mathrm{pg}$ Hex- $\lambda$ VP2 RNA and 100 pg nuclear $\beta$-galactosidase per blastomere. The schematic diagram in the lower right-hand corner indicates the stage, position and nature of each injection (red, Hex- $\lambda$ VP2 alone; yellow, $\beta$-galactosidase alone; orange, Hex- $\lambda$ VP2 and $\beta$-galactosidase; lime green, $\beta$-galactosidase, Hex- $\lambda$ VP2 and Hex). (A) Stage 36 control tadpole. $(B, C) H e x-\lambda V P 2$ RNA injected into two dorsal blastomeres at the 4-cell stage. (D) Hex- $\lambda$ VP2 RNA injected into two dorsal-vegetal blastomeres at the 8-cell stage. (E) $\beta$-galactosidase RNA injected into a single ventral-vegetal blastomere at the 8-cell stage. $(\mathrm{F}-\mathrm{H}) \beta$-galactosidase and Hex- $\lambda$ VP2 RNA injected into a single ventral-vegetal blastomere at the 8 -cell stage. (I-J) $\beta$-galactosidase and Hex- $\lambda$ VP2 RNA injected into two dorsal blastomeres at the 4-cell stage. (K) $\beta$-galactosidase and Hex- $\lambda$ VP2 RNA injected two dorsal animal blastomeres at the 8-cell stage. (L) $\beta$-galactosidase, Hex- $\lambda$ VP2 and Hex RNA injected into two dorsal blastomeres at the 8-cell stage. (M,N) $\beta$ galactosidase and Hex- $\lambda$ VP2 RNA injected into two dorsal-vegetal blastomeres at the 8-cell stage. Frequencies for the phenotypes described above are listed in Table 1. For dorsal animal injections, $62 \%$ of cases were normal, $10 \%$ showed anterior truncations, $10 \%$ were cyclopic and $20 \%$ had non-specific gastrulation defects $(n=21)$.

Table 1. Phenotypic frequencies in response to injection of Hex- $\lambda$ VP2 derivatives

\begin{tabular}{|c|c|c|c|c|c|c|c|c|c|c|c|c|c|c|c|}
\hline \multirow[b]{3}{*}{ RNA } & \multicolumn{5}{|c|}{ Dorsal phenotypes $(\%)$} & \multicolumn{6}{|c|}{ Marker expression in ventral-lateral injections } & \multicolumn{4}{|c|}{ Ventral vegetal phenotypes (\%) } \\
\hline & \multirow[b]{2}{*}{$n$} & \multirow{2}{*}{$\begin{array}{l}\text { Anterior } \\
\text { truncation }\end{array}$} & \multirow[b]{2}{*}{ Cyclopic } & \multirow{2}{*}{$\begin{array}{c}\text { Gastrulation } \\
\text { defect }\end{array}$} & \multirow[b]{2}{*}{ Normal } & \multicolumn{2}{|r|}{ Gsc } & \multicolumn{2}{|r|}{ Chordin } & \multicolumn{2}{|r|}{ Noggin } & \multirow[b]{2}{*}{$n$} & \multirow[b]{2}{*}{ Normal } & \multirow[b]{2}{*}{ Mild } & \multirow[b]{2}{*}{ Severe } \\
\hline & & & & & & $n$ & Ectopic (\%) & $n$ & Ectopic (\%) & $n$ & Ectopic (\%) & & & & \\
\hline Hex $\lambda$ VP2 & 160 & 44 & 41 & 9 & 6 & 52 & 71 & 36 & 78 & 39 & 0 & 38 & 5 & 37 & 63 \\
\hline $\operatorname{Hex}(\mathrm{N} 186 \mathrm{P}) \lambda \mathrm{VP} 2$ & 42 & 5 & 0 & 19 & 76 & 20 & $10^{* *}$ & nd & - & nd & - & nd & - & - & - \\
\hline $\operatorname{Hex}(\mathrm{N} 186 \mathrm{~K}) \lambda \mathrm{VP} 2$ & 22 & 18 & 23 & 59 & 0 & 16 & $6^{* *}$ & nd & - & nd & - & nd & - & - & - \\
\hline $\begin{array}{l}\text { Hex } \lambda \text { VP2:Hex } \\
1: 1\end{array}$ & 18 & 17 & 56 & 0 & 27 & nd & - & nd & - & nd & - & nd & - & - & - \\
\hline $\begin{array}{l}\text { Hex } \lambda \text { VP2:Hex } \\
1: 2\end{array}$ & 33 & 6 & 18 & $76^{*}$ & 0 & nd & - & nd & - & nd & - & nd & - & - & - \\
\hline $\begin{array}{l}\text { Hex } \lambda \text { VP2:Hex } \\
1: 4\end{array}$ & nd & - & - & - & - & nd & - & nd & - & nd & - & 20 & 50 & 45 & 5 \\
\hline $\begin{array}{l}\text { Hex } \lambda V \mathrm{VP} 2 \\
\Delta 56\end{array}$ & nd & - & - & - & - & 10 & 0 & 11 & $18^{* *}$ & 7 & 0 & nd & - & - & - \\
\hline
\end{tabular}



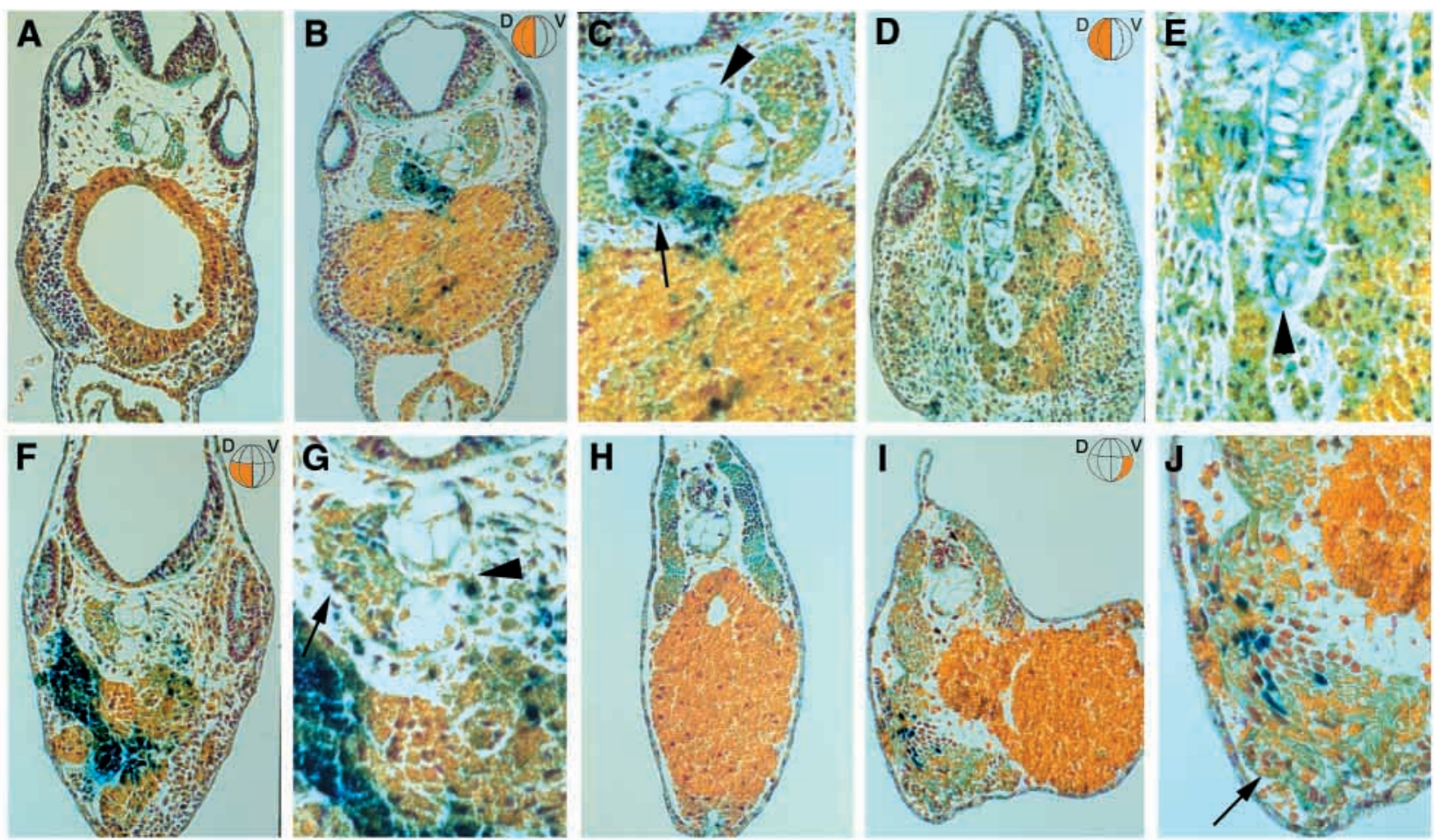

Fig. 3. Hex- $\lambda$ VP2 induces dorsal mesoderm. Transverse sections through embryos described in Fig. 2. Dark blue stain is $\beta$-galactosidase lineage tracer and sections are stained with Feulgen, Light Green and Orange G. Muscle tissue and somite are denoted by an arrow, and notochord by an arrowhead. The type of injection is depicted in cartoon form in the upper right hand corner of each panel as in Fig. 2. (A) Control embryo at the hindbrain level, (B-E) dorsal injections, (F-G) dorsal-vegetal injection, (H) control embryo, (I-J) ventral-vegetal injection. The images in $\mathrm{C}, \mathrm{E}, \mathrm{G}$ and $\mathrm{J}$ are higher magnification views of sections in $\mathrm{B}, \mathrm{D}, \mathrm{F}$ and $\mathrm{I}$.

activators known, but as a result it can be lethal in yeast and mammalian cells as a result of the phenomenon known as squelching (reviewed by Ptashne, 1992). The modular forms of the minimal amino terminal domain of VP16 described in this study appear not to have toxic side effects as overexpression of a point mutation in Hex fused to $\lambda$ VP2 has no phenotype in Xenopus (see below) and over-expression of Hex- $\lambda$ VP2 at levels tenfold higher than those used in this study did not kill animal cap explants (data not shown). However, to avoid potential problems as a result of squelching, all in vivo experiments in this paper use Hex- $\lambda$ VP2 rather than Hex$\lambda$ VP4.

\section{Mis-expression phenotypes of Hex and Hex- $\lambda$ VP2}

To assess the role of repression by Hex on anterior patterning, RNA encoding Hex- $\lambda$ VP2 was injected into Xenopus embryos at the 4- and 8-cell stages (Table 1). Fig. 2 shows the results of these experiments. Embryos shown in the top panel of Fig. 2 were injected on the dorsal side at the 4-cell stage (B,C; also see $\mathrm{I}, \mathrm{J}$ for co-injection with $\beta$-galactosidase lineage tracer) and 8 -cell stage $(\mathrm{D}$; also see $\mathrm{L}, \mathrm{M}, \mathrm{N}$ for co-injection with a $\beta$ galactosidase lineage tracer). Complete anterior truncation occurred in $44 \%$ of injected embryos while the cyclopic phenotype shown in Fig. 2D occurred with a frequency of $41 \%$. Truncations occurred just anterior of the mid-hind brain boundary as demonstrated by in situ hybridization with Krox 20 and Engrailed (data not shown). These embryos represent the range of phenotypes described in Table 1. Transverse sections at the level of the hindbrain of injected embryos (Fig.
3A-G) show expanded somites and duplication of the notochord. Use of a $\beta$-galactosidase lineage tracer indicates that this expansion of dorsal structures occurs in both a cellautonomous and a cell-non-autonomous manner (Fig. 3C,E,G).

The basis for the expansion of dorsal structures and loss of anterior tissues became apparent when RNA encoding Hex$\lambda \mathrm{VP} 2$ was injected on the ventral side at the 8-cell stage (Fig. 2E-H). In these embryos the range of phenotypes includes ectopic induction of disorganized muscle (sections through embryos such as that shown in Fig. 2G, see for example Fig. 3I), partial secondary axis formation (Fig. 2F), or dorsalisation (Fig. 2H). Sections through ventral-vegetally injected embryos show cell-non-autonomous induction of muscle (Fig. 3H-J). Fig. 2 also shows that when similar concentrations of RNA encoding Hex- $\lambda$ VP2 were injected into dorsal-animal blastomeres at the 8-cell stage, there was relatively little anterior phenotype (Fig. $2 \mathrm{~K}$ ), suggesting that at these RNA concentrations, Hex- $\lambda$ VP2 has little effect when expressed in blastomeres that are fated to become anterior ectoderm. This is consistent with molecular marker analysis which shows that induction of markers in response to Hex- $\lambda$ VP2 in the ectoderm is not as dramatic as in other tissues (data not shown). When wild-type Hex was co-injected with Hex- $\lambda$ VP2 in ventral vegetal blastomeres, rescue of the Hex- $\lambda$ VP2 phenotype was observed (Table 1). When wild-type Hex was co-injected with Hex- $\lambda$ VP2 into dorsal blastomeres at a 1:1 ratio we observed a low frequency of rescue (for example, Fig. $2 \mathrm{~L}$ ) and at a 2:1 ratio we observed a defect in gastrulation as a result of Hex over-expression (Table 1, see below). The pronounced defect 

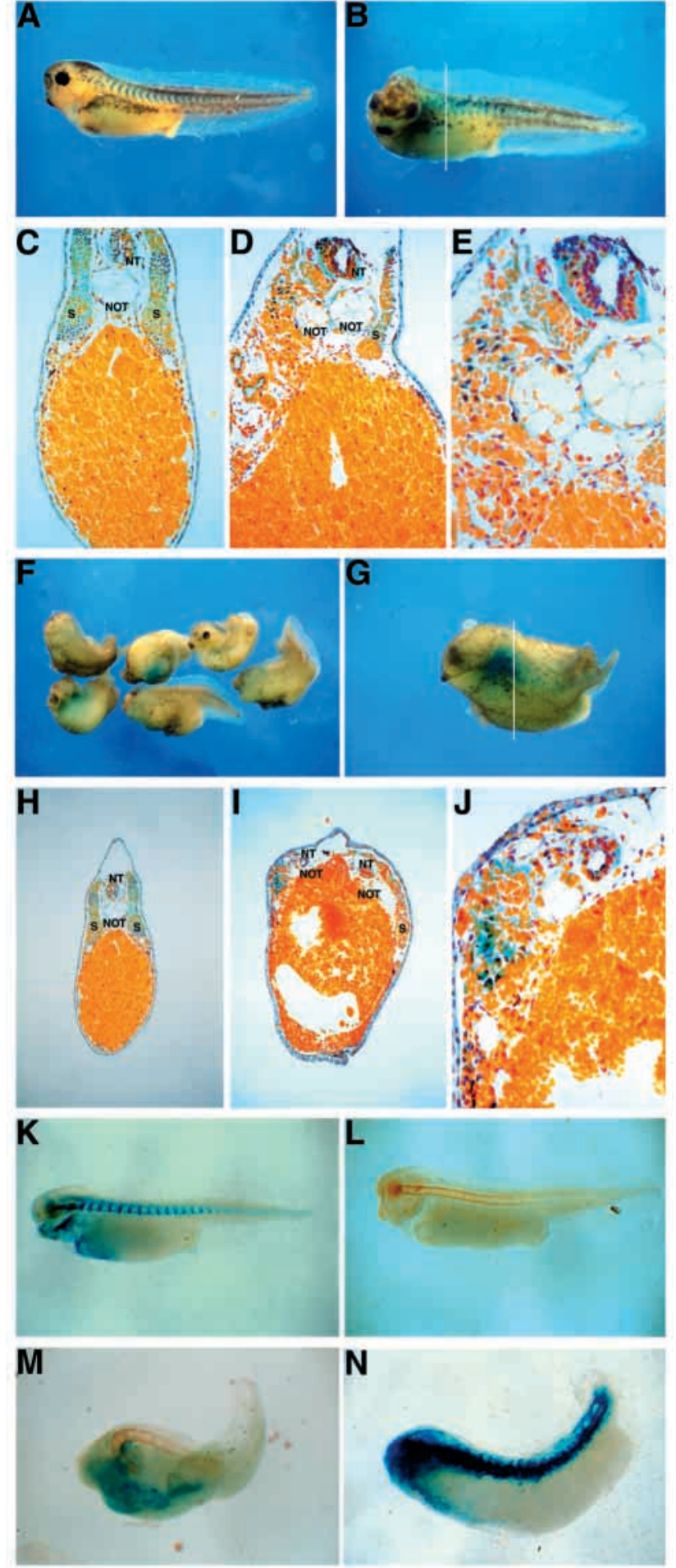

in gastrulation (see below), which is a consequence of Hex over-expression on the dorsal side, makes it difficult to determine a ratio of Hex:Hex- $\lambda$ VP2 at which there are no phenotypic consequences.

The induction of dorsal mesoderm by Hex- $\lambda$ VP 2 suggests that wild-type Hex defines an anterior state by suppressing the induction and/or propagation of dorsal mesoderm (or trunk organiser). To determine the consequences of Hex over-expression on the dorsal side under conditions in which
Fig. 4. Phenotypes produced by Hex RNA injection. Hex RNA ( 250 pg) was co-injected with a $\beta$-galactosidase lineage tracer into a single dorsal or a single dorsal-vegetal blastomere. (A) Control embryo injected with $\beta$-galactosidase lineage tracer. (B) Embryo injected with RNA encoding both Hex and $\beta$-galactosidase. $(\mathrm{C}, \mathrm{H})$ Sections through control embryos at comparable levels to D,E and I,J, respectively. Injection of Hex RNA caused either bulges around the mid-line (B) or loss of anterior and posterior axial structures (F,G). (D,E) Sections through the embryo in B showing disruption of the somite on the injected side (indicated by blue labelled cells), a yolky endoderm-like extension and induction/expansion of notochord. $(\mathrm{I}, \mathrm{J})$ Sections through the embryo in $\mathrm{G}$ showing a second neural tube in addition to disruption of the somite and induction of notochord on the injected side. Identification of notochord is confirmed by examination of serial sections. Antibody staining with the notochordspecific MZ15 shows these effects to be non-cell autonomous. (K) Cleared embryo injected with RNA encoding $\beta$-galactosidase, and stained with MZ15. (I) Embryo stained solely with MZ15 (orange); comparison with $\mathrm{K}$ reveals co-localisation of $\beta$ galactosidase and MZ15 staining. (M) Embryo injected with RNA encoding Hex has an enlarged notochord (orange) distinct from the population of Hex-injected cells (blue). (N) Embryo injected with Hex- $\lambda$ VP2 and stained with MZ15. The enlarged notochord shown in Fig. 3 is obscured by labeled cells which occupy axial positions. S, somite; NT, neural tube; NOT, notochord. After 4-cell dorsal injections $(n=32), 33 \%$ showed a severe phenotype (Fig. 4F) and $66 \%$ a mild phenotype (Fig. 4B). After 8-cell dorsal injections $(n=32), 19 \%$ showed a severe phenotype (Fig. 4F) and $81 \%$ a mild phenotype (Fig. 4B).

gastrulation can occur, we targeted Hex RNA either into a single dorsal blastomere at the 4-cell stage or a single dorsal vegetal blastomere at the 8 -cell stage. Fig. 4 shows that overexpression of Hex under these conditions leads either to mild distortions of the embryonic axis (Fig. 4B) or to a more extreme phenotype, which displays defects in both anterior and posterior structures (Fig. 4F,G). Despite the anterior defects displayed in the more extreme phenotypes all embryos appear to have an enlarged head with a dramatically shortened A-P axis (Fig. 4F). Histological analysis of injected embryos shows that anterior neural defects notwithstanding, a large proportion of injected embryos have enlarged cement glands (data not shown). Consistent with the results obtained with RNA encoding Hex- $\lambda$ VP2, injection of Hex RNA leads to disruption of somitic tissue on the injected side and replacement of somite with a yolky, endoderm-like tissue, extending up from the endodermal yolk mass (Fig. 4D,I). Surprisingly, immediately adjacent to the cells injected with Hex, expanded or ectopic dorsal structures such as notochord are formed (Fig. 4E,J). Double staining using the $\beta$-galactosidase lineage tracer and the notochord-specific MZ15 antibody (Smith and Watt, 1985) (orange in Fig. 4K-N) shows how this result contrasts with the ectopic induction of dorsal mesoderm observed in response to injection of Hex- $\lambda$ VP2. The effects of Hex are cell non-autonomous and injected cells appear to segregate from the expanded notochord (Fig. 4M) whereas the expanded notochord induced by Hex- $\lambda$ VP2 is populated by injected cells; the induction of axial mesoderm in response to Hex- $\lambda$ VP2 is therefore cell autonomous (Fig. 4N). Thus Hex- $\lambda$ VP2-injected cells appear to form trunk organiser derivatives whereas Hex-injected cells have the capacity to induce dorsoanterior structures. 


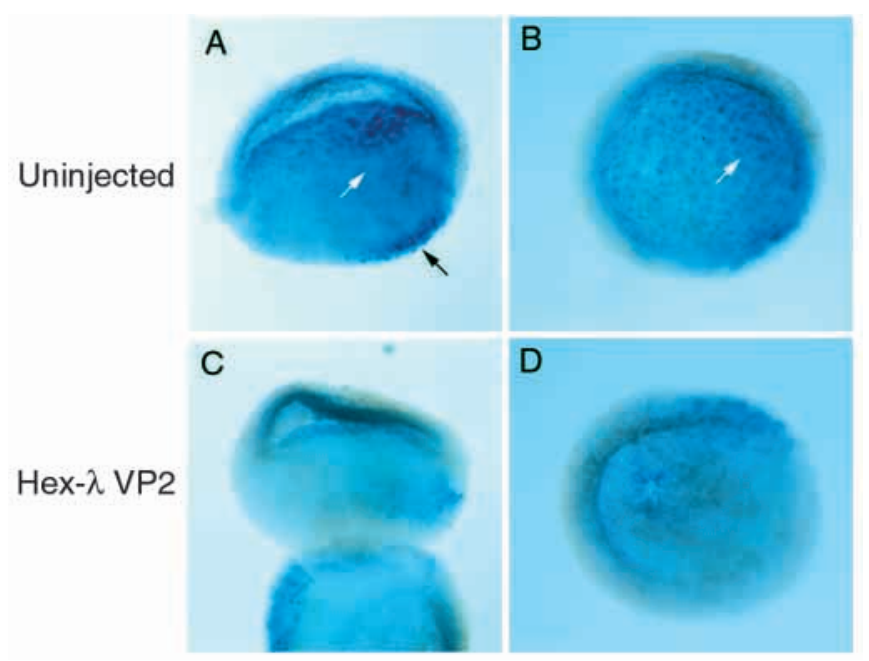

Fig. 5. Injection of Hex- $\lambda$ VP2 RNA inhibits Cerberus expression. (A) Lateral view and (B) animal view of early gastrula stage 10-10.5 uninjected embryo. (C) Lateral view and (D) animal view of embryo injected with Hex- $\lambda$ VP2 RNA. White arrows indicates the deep expression domain of Cerberus and the black arrow the suprablastoporal domain. Embryos were injected with 25 pg of Hex$\lambda$ VP2 RNA into both dorsal blastomeres at the 4-cell stage. All embryos were cleared in Murray's agent to visualize deep tissues.

\section{Hex is a suppressor of dorsal mesoderm that is necessary for the maintenance of anterior endoderm}

Correlating with the anterior truncations caused by injection of Hex- $\lambda$ VP2 is a reduction in the anterior endoderm domain marked by expression of Cerberus. At the early gastrula stage, Cerberus is expressed in two domains, an involuting suprablastoporal population of anterior mesendoderm (Fig. $5 \mathrm{~A}$, black arrow) and a population of deep, non-involuting endoderm cells, extending from the floor of the blastocoel into the vegetal hemisphere (Fig. 5A,B, white arrows). Injection of RNA encoding Hex- $\lambda \mathrm{VP} 2$ on the dorsal side of the embryo causes a reduction in Cerberus expression (Fig. 5C,D). The effect is particularly pronounced in the deep, non-involuting endoderm population, which has been compared to the AVE of the mouse. Since Hex is a transcriptional repressor in vitro, and Hex- $\lambda$ VP2 functions as an activator, any inductive relationship between Hex and Cerberus must be indirect.

The ability of Hex- $\lambda$ VP2 to induce trunk organiser or dorsal mesoderm is also reflected in induction or expansion of certain dorsal mesodermal markers early in development. At gastrulation stages (10-11), Chd (Sasai et al., 1994) and Noggin (Smith et al., 1993) are expressed in dorsal mesoderm and function to antagonize BMP4 signaling (McMahon et al., 1998; Piccolo et al., 1996). BMP4 at this stage is expressed in the ventral marginal zone and the ectoderm (HemmatiBrivanlou and Thomsen, 1995). Fig. 6A shows that injection of RNA encoding Hex- $\lambda$ VP2 induced $C h d$ and inhibited $B M P 4$ expression. However, in contrast to $C h d$, expression of Noggin was not affected by Hex- $\lambda$ VP2 (Fig. 6A). Consistent with these results, injection of RNA encoding Hex into dorsal blastomeres inhibited Chd expression, but not Noggin (Fig. 6B).

Together, our results suggest that Hex suppresses trunk organiser formation. These observations are supported by axis duplication experiments with a dominant negative form of the
A

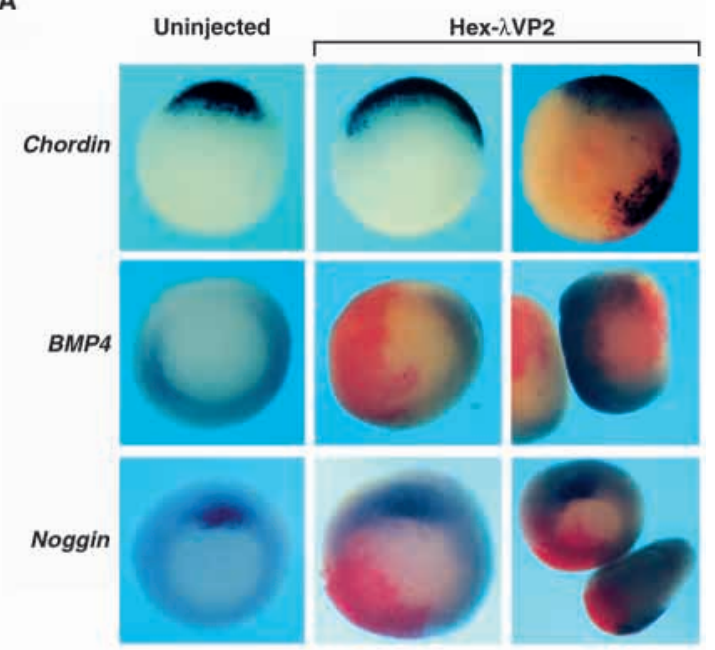

B

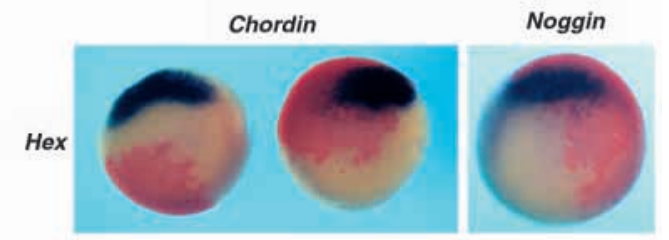

Fig. 6. Induction of dorsal mesoderm by Hex- $\lambda \mathrm{VP} 2$ and suppression by Hex. (A) Injections of RNA encoding Hex- $\lambda$ VP2 induces ectopic $C h d$, causes down-regulation of BMP4 and has no effect on Noggin. (B) Injections of RNA encoding Hex inhibit expression of $C h d$ and not Noggin. Embryos were co-injected with synthetic RNA and a fluorescent dextran lineage trace. In situ hybridization for the indicated marker is in blue and the lineage tracer is stained in red. All embryos were fixed at stage 10-10.5 and are orientated with dorsal upwards. The majority of injections were done at the 4- or 8-cell stage into ventral lateral blastomeres or in one case, at the top of (A), throughout the dorsal side (second panel from left). In B all embryos were injected at the 4-cell stage into either a single dorsal or ventral blastomere. Embryos were injected with either 25 pg of Hex- $\lambda$ VP2 RNA or 250 pg Hex RNA.

BMP receptor. Ventral-vegetal injection of this construct induces partial secondary axis formation in injected embryos (Ishikawa et al., 1995), and co-injection of Hex suppressed the axis duplication activity of the dominant negative BMP receptor (data not shown).

Another marker of dorsal mesoderm, and one of the earliest markers of organiser tissue, is the homeobox gene Gsc. In ectopic expression experiments Gsc apparently has the opposite effect to that of Hex, as Gsc will induce Chd and not Noggin (Sasai et al., 1994). Like Chd and Noggin, Gsc is expressed on the dorsal side of the Xenopus gastrula (Cho et al., 1991). Expression of RNA encoding Hex- $\lambda$ VP2 throughout the dorsal region of the embryo led to an expansion of the endogenous Gsc domain (Fig. 7A). Similarly, injection of RNA encoding Hex- $\lambda$ VP2 into ventrolateral locations led to ectopic Gsc expression in a cell-autonomous fashion (Fig. 7A). Hex$\lambda$ VP2 expression also led to induction of Gsc in animal cap explants (Fig. 7C). The reciprocal experiment of targeting wild-type Hex to either the entire dorsal side or to half of the 
endogenous domain of Gsc (to allow gastrulation to proceed) caused down-regulation of Gsc (Fig. 7B), and injection of wildtype Hex RNA inhibited induction of Gsc in animal cap explants treated with activin (Fig. 7C). Animal cap explants treated with activin undergo convergent extension or gastrulation-like movements (Symes and Smith, 1987). Injection of Hex inhibited these movements, while injection of Hex- $\lambda$ VP2 induced them (data not shown).

\section{Direct interaction of Hex with the Goosecoid promoter}

The effect of Hex on Cerberus expression appears indirect. However, Hex is a transcriptional repressor and in misexpression experiments it suppresses Gsc in a cell-autonomous manner, suggesting that Hex might regulate Gsc directly. Fig. 8 shows that Hex- $\lambda$ VP2 recognizes the Gsc promoter in cell culture. Examination of the Gsc activin and Wnt response elements (Watabe et al., 1995) revealed the presence of a potential Hex binding site between nucleotides -122 and -115 (CATTAAAT); this is based on identity (at 7 out of 8 positions) to the site defined previously by Crompton et al. (1992) in binding site selection experiments. Fig. 8A shows an experiment in which Hex- $\lambda$ VP2 was co-transfected with different Luciferase reporter genes containing fragments of the Gsc promoter positioned upstream of the Gsc minimal TATA box. Co-transfection of a plasmid expressing Hex- $\lambda$ VP2 over a range of concentrations led to a fourfold induction of a 300 bp fragment of the Gsc promoter. This region is sufficient to mediate responses to both Wnt and activin signalling in animal cap explants (Watabe et al., 1995). A $5^{\prime}$ deletion of this promoter element containing sequences from -142 nucleotides upstream of the start of transcription also responded to Hex$\lambda$ VP2, but a smaller fragment comprising nucleotides from position -37 did not. Full induction of the Gsc promoter in response to Hex- $\lambda$ VP2 appears to require the putative Hex binding site at position -122 .

\section{Specificity of Hex- $\lambda$ VP2 activity in vivo and in vitro}

Hex is a member of the Antennapedia /Ftz homeodomain subclass, having an asparagine at position 9 in the recognition helix (Crompton et al., 1992). Mutation of this position to a lysine has been shown in two cases to alter the class of DNA site recognized by the homeodomain to that of Bicoid (Schier and Gehring, 1992; Treisman et al., 1989; Wilson et al., 1993). Alteration of this position in Hex (homeodomain position 50) to either lysine (HexN186K), which alters the specificity of the Hex homeodomain, or proline (HexN186P), which should break the recognition helix, yields fusion proteins that are unable to induce transcription of the Gsc promoter in transient transfections (Fig. 8B). Similar results are obtained when these proteins are expressed fused to GAL4 (also see Fig. 8B), creating the tripartite proteins GAL4-Hex(N186P)- $\lambda$ VP2 and GAL4-Hex(N186K)- $\lambda$ VP2. Thus, co-transfection of DNA encoding GAL4-Hex- $\lambda$ VP2 with the Gsc promoter resulted in a tenfold induction of transcription, whereas co-transfection of DNA encoding either GAL4-Hex(N186P)- $\lambda$ VP2 or GAL4Hex(N186K)- $\lambda$ VP2 with the same reporters over a tenfold range of concentrations resulted in no detectable induction. However, when DNA encoding GAL4-Hex(N186P)- $\lambda$ VP2 or GAL4-Hex(N186K)- $\lambda$ VP2 is co-transfected along with reporter genes containing GAL4 sites (Fig. 8C), they are at
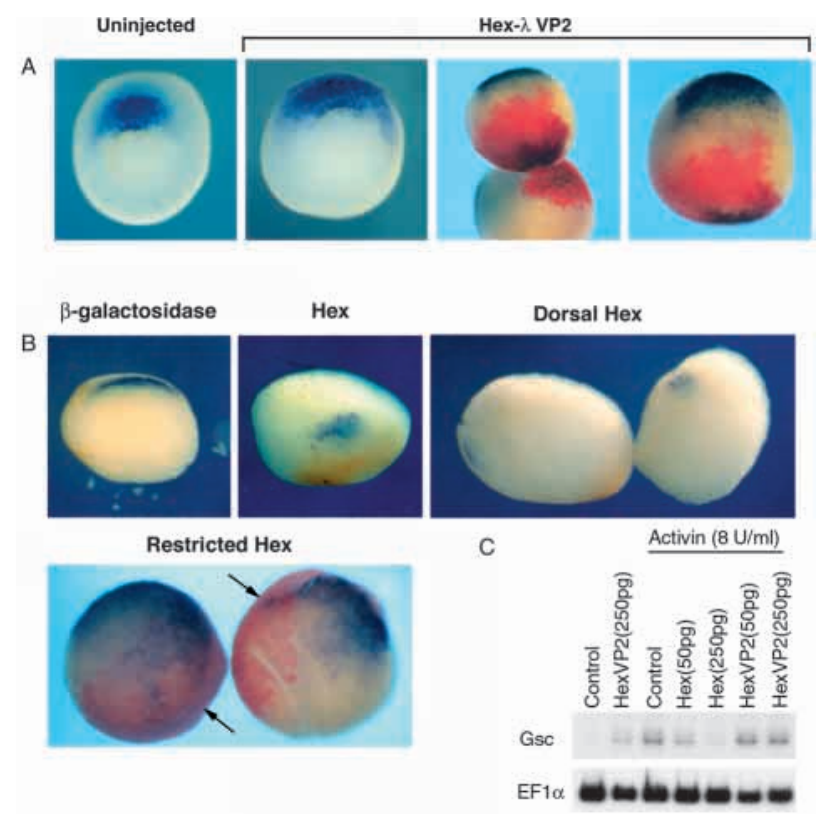

Fig. 7. Regulation of Goosecoid by Hex. (A) Injection of Hex- $\lambda$ VP2 RNA induces ectopic Gsc expression. Gsc expression is in blue and the fluorescent dextran lineage tracer is in red. (B) Injection of Hex RNA leads to a reduction in Gsc expression. Equivalent

concentrations of Hex or $\beta$-galactosidase RNA (1 ng), as a negative control, were injected into embryos at the 2-cell stage, on the dorsal side at 4-cell stage or, in the bottom panel, 500 pg of Hex RNA was injected into either a single dorsal or a single ventral blastomere at the 4-cell stage (the arrow indicates the location of the injection relative to the endogenous Gsc domain). As in (A), in situ hybridization for Gsc is in blue and the lineage tracer is red. The stage and orientation of embryos is as Fig. 6. (C) Hex and Hex- $\lambda$ VP2 activity in animal cap explants. Hex RNA and $E F-1 \alpha$, as an internal control, were detected by RT-PCR. The bar over the second set of lanes indicates that the animal cap explants were cultured in the presence of 8 units $/ \mathrm{ml}$ of activin, to induce Gsc. Embryos were injected with the same amount of RNA as those in Fig. 5 unless otherwise indicated.

least as active as GAL4-Hex $\lambda$ VP2, demonstrating that these mutations specifically interfere with the ability of the Hex homeodomain to recognize its natural site, rather than by disrupting the overall activity or stability of these fusion proteins.

When assayed by electrophoretic mobility shift assays, in vitro translated Hex, Hex- $\lambda$ VP2 and Hex(N186K)- $\lambda$ VP2, but not $\operatorname{Hex}(\mathrm{N} 186 \mathrm{P})-\lambda \mathrm{VP} 2$, bound to the putative Hex site CATTAAAT (data not shown). The ability of $\operatorname{Hex}(\mathrm{N} 186 \mathrm{~K})$ to bind this site was surprising because ES cell transfection assays show a tenfold reduction in specific recognition of the Gsc promoter by the N186K mutation. However, the transient transfection experiments were done in ES cells and these cells may contain additional components that affect Hex-DNA binding. The Gsc reporter constructs may also contain additional DNA sequences involved in Hex-DNA binding.

Fig. 8 also shows that progressive truncation of the amino terminus of Hex prevents Hex from recognizing the Gsc promoter in ES cell transient transfection. Removal of either the amino terminal Engrailed homology domain (a sequence conserved in a large number of transcription factors and 
necessary for repression by Engrailed; Smith and Jaynes 1996) or the entire amino terminus up to the homeodomain, leads to a loss of Hex DNA binding activity in transient transfection assays. Interestingly the binding site selection experiments performed with Hex were done with a protein fragment missing all coding sequence amino terminal of the homeodomain (Crompton et al., 1992) and this may explain why this isolated sequence appears insufficient for us to detect sequence specific DNA binding in vitro.

The phenotypes obtained when RNA encoding these mutant forms of Hex- $\lambda$ VP2 was injected into Xenopus embryos are summarized in Table 1. Mutation K186P, in the context of Hex- $\lambda$ VP2, led to a complete loss of phenotype in response to RNA injection, while mutation Q186K resulted in defects in gastrulation movements which were distinct from the phenotype induced by wild-type Hex- $\lambda$ VP2. Neither fusion protein activated ectopic Gsc (Fig. 9 and Table 1) and neither Gsc nor Chd was significantly upregulated in response to ectopic expression of $\Delta 56 \mathrm{Hex}-\lambda \mathrm{VP} 2$ (Table 1).

\section{The expression patterns of Gsc and Hex reveal independent domains established prior to gastrulation}

At first sight, the observation that induction of Gsc by Hex- $\lambda$ VP2 is associated with loss of anterior structures is surprising, because Gsc is expressed in anterior mesendoderm and anterior organiser derivatives (Cho et al., 1991). We note, however, that Hex and Gsc occupy distinct expression domains from late blastula to mid gastrula stages, with Hex being expressed more anteriorly by late gastrula stages (Fig. 10). Inspection of published data and our own in situ analyses (not shown) suggest the same is true in the mouse.

Fig. 8. Hex recognises the Goosecoid promoter in ES cells. (A) Deletion analysis of the activin response element in the Gsc promoter by transient transfection with DNA encoding Hex- $\lambda$ VP2. (B) Activity of Hex$\lambda$ VP2, GAL4-Hex- $\lambda$ VP2 and different point mutations in Hex from the Gsc promoter. (C) Activity of GAL4Hex- $\lambda$ VP2 and point mutations from 5 GAL4 binding sites. The level of activation of Gsc is fold induction. The data represented here is based on multiple experiments in which the basal levels have all been normalized to 1 . Error bars represent standard deviations. The overall activity in $\mathrm{C}$ is the total level of transcription from this promoter. Each number represents an average of multiple independent transfections with the error bar representing the standard deviation. Arrows indicate transfection of increasing amounts of DNA encoding the protein indicated. In A and B 200, 400 or 800 ng of a plasmid expressing the indicated Hex derivative were cotransfected with $100 \mathrm{ng}$ of the indicated reporter. In $\mathrm{C}$ a range of concentrations of GAL4-Hex- $\lambda \mathrm{VP}$ was transfected (100-600 ng), while subsets of these values (one low point and one high point) were used for all the other indicated Hex derivatives.

B

C

\section{DISCUSSION}

In this paper we show that Hex is a transcriptional repressor required for the establishment of anterior identity and that it has the capacity to suppress markers associated with the organiser. Conversion of Hex to an activator by fusion to modules of the VP16 activation domain results in a chimeric
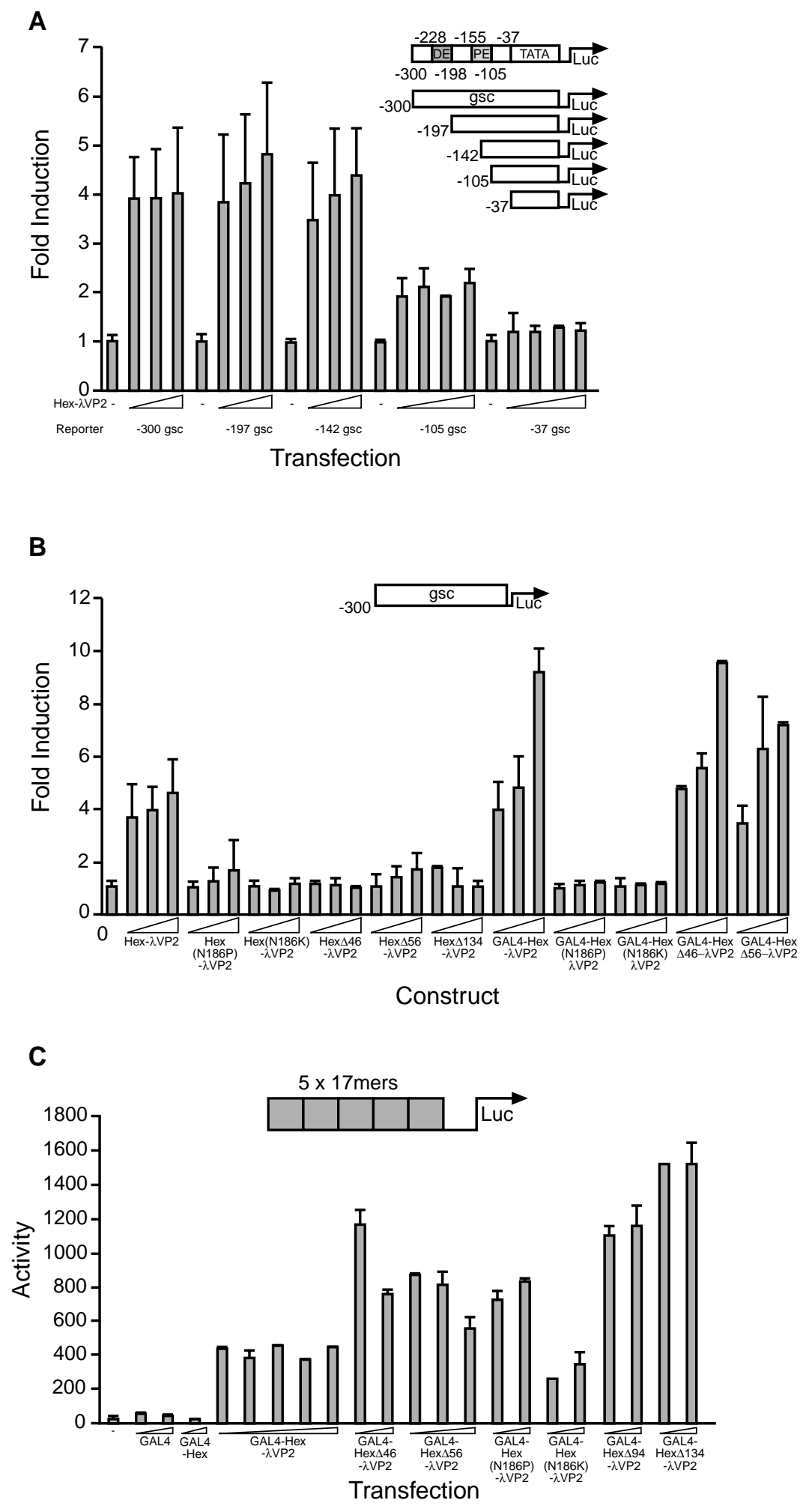


\section{J. M. Brickman and others}

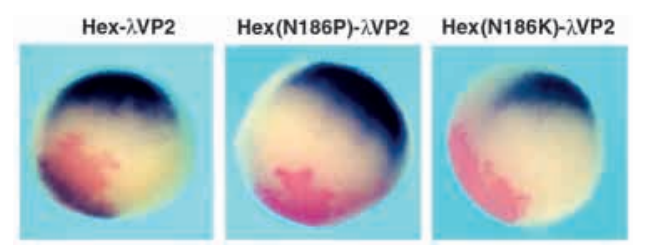

Fig. 9. Hex- $\lambda$ VP2 but not Hex(N186P)- $\lambda$ VP2, or Hex(N186K)- $\lambda$ VP2 induces Gsc. All injections were $25 \mathrm{pg}$ of RNA in a ventral position at the 16-cell stage.

protein that in mis-expression experiments produces anterior truncations, induction of trunk dorsal mesoderm and induction of early mesodermal markers such as Gsc and Chd, while inhibiting the anterior endoderm marker Cerberus. Transient transfection experiments in ES cells indicate that this chimeric protein can recognize sequences in the activin response element of the Gsc promoter and that mutations in Hex- $\lambda$ VP2 which affect recognition of Gsc in ES cells either alter or eliminate the phenotype of Hex- $\lambda$ VP2 RNA injection in Xenopus embryos.

\section{Structure-function analysis of Hex and the generation of dominant negative proteins}

The Hex- $\lambda$ VP2 molecule is a potent inducer of target gene expression. When the reiterated VP16 modules described in this study were tested as GAL4 fusions by both transient transfection and in yeast nuclear extracts in vitro, they were shown to behave synergistically (Emami and Carey, 1992; Ohashi et al., 1994). Moreover, in both yeast and mammalian cells, the $\lambda$ repressor linker was shown to potentiate the activity of these modules without having intrinsic transcriptional activation function (Emami and Carey, 1992; Ohashi et al., 1994). In the case of Hex, the positioning of a flexible linker between the Hex coding sequence and transcriptional activation domains may avoid steric constraints generated when an activation domain is fused to a transcriptional repressor. As a result, we have been able to modify the activity of Hex without removing the endogenous repression domain. This has the advantage that there is little risk of perturbing additional structural motifs involved in promoter recognition that may lie outside the homeodomain. For example, in both transient transfections and in vivo ectopic expression experiments, we observed a defect in DNA binding by Hex$\lambda$ VP2 derivatives lacking portions of the Hex amino terminus. Our smallest 46 amino acid deletion removes the conserved Engrailed homology domain 1 which, in Drosophila Gsc, mediates interactions between Gsc and the homeodomain of Otd (Mailhos et al., 1998). In the context of Hex this domain might therefore mediate homo-dimerisation or interaction with other DNA binding factors that mediate promoter specificity in the embryo.

\section{Negative regulation and anterior induction}

Recent models of anterior patterning suggest that the induction of head structures involves the simultaneous inhibition of TGF $\beta$ and Wnt signals (Piccolo et al., 1999; Glinka et al., 1997). For example, induction of heads by Cerberus is due to its capacity to inhibit signalling by BMP4, Xnr1 and XWnt8 (Piccolo et al., 1999), and indeed head formation can also be observed following simultaneous and independent inhibition of

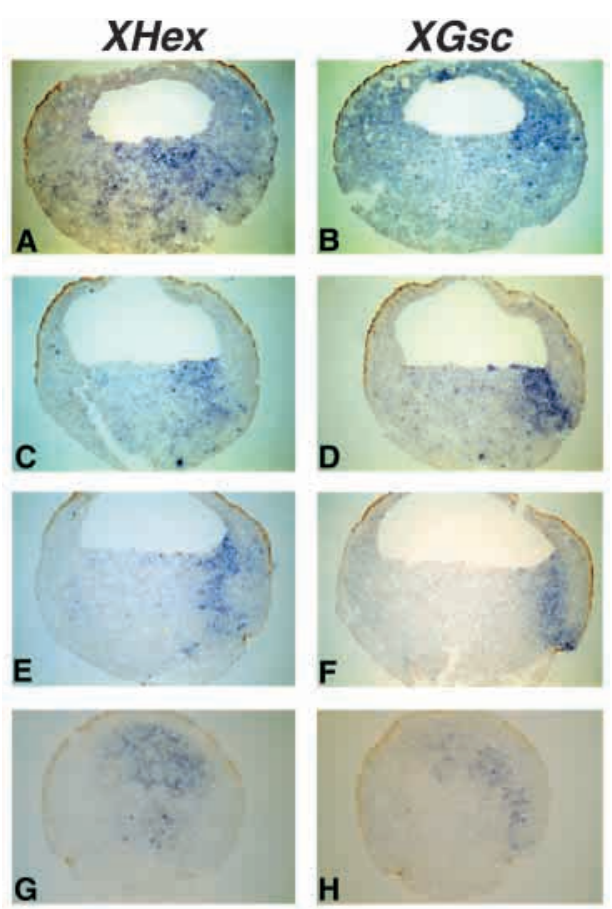

Fig. 10. Spatial expression patterns of Gsc and Hex during gastrulation. In situ hybridization in near-adjacent sections of Xenopus embryos with probes to Hex and Gsc. A,C,E and G are stained for Hex and B,D,F,H are stained for Gsc. Embryo in A and B is stage 9, embryo in $C$ and $D$ is stage 10-10.5, embryo in $E$ and $F$ is stage 10.5-11, embryo in $\mathrm{G}$ and $\mathrm{H}$ is stage 11-11.5. The sections in $\mathrm{G}$ and $\mathrm{H}$ go through the dorsal side at an angle that is imperfectly transverse. Thus anterior is at the top and dorsal extends down to the right.

these three signalling pathways (Glinka et al., 1997). These results suggest that the role of Cerberus and, more generally, of anterior endoderm, is to suppress formation of the trunk tissue that is normally induced by TGF $\beta$ and Wnt signals. Our experiments indicate that Hex, a transcriptional repressor, plays a role in this process.

Comparison of the phenotypes observed in our ectopic expression experiments with those following Cerberus overexpression suggests that Hex and Cerberus may act in similar pathways. For example, ventral marginal zone explants injected with the inhibitory molecule Cerberus develop headlike structures lacking any evidence of somites and notochord (Bouwmeester et al., 1996; Piccolo et al., 1999). In what may be the reciprocal experiment we observe induction of additional somites and notochord in response to Hex- $\lambda$ VP2.

Our findings that Hex is a transcriptional repressor, and that Hex- $\lambda$ VP2 inhibits Cerberus expression, suggest that Hexmediated induction of Cerberus occurs indirectly through the suppression of factors that normally repress Cerberus in nonanterior endoderm. This indirect induction may explain why Hex induction of Cerberus is highly context dependent, occurring only when Hex is expressed in the endoderm (Jones et al., 1999). It contrasts with the more promiscuous induction of Cerberus by other factors such as Sox 17 and Mixer (Henry and Melton, 1998; Hudson et al., 1997).

Ectopic expression of Hex RNA in zebrafish has been recently shown to expand the expression of dorsal markers 
such as Chordin (Ho et al., 1999), whereas our data show that Hex suppresses both Chordin and Gsc. We can best explain this discrepancy by suggesting that the expansion of chordin in zebrafish reflects the non cell-autonomous effects of Hex, which include, in Xenopus, the induction of dorsal tissues (Fig. 4). In Xenopus it is easier to distinguish between cell autonomous and non cell-autonomous effects, because it is possible to target RNA expression to particular regions of the embryo. Consistent with the idea that Hex plays similar roles in Xenopus and zebrafish, injection of RNA encoding Hex$\lambda$ VP2 into zebrafish embryos causes dorsalisation at high concentrations and anterior truncation at lower concentrations (C. Houart and S. Wilson, personal communication).

Finally, we note that the phenotypes observed in response to mis-expression of Hex resemble those obtained following expression of dominant negative Wnt molecules (Deardorff et al., 1998; Hoppler et al., 1996; Itoh and Sokol, 1999) or of Wnt antagonists (Glinka et al., 1998; Leyns et al., 1997; Salic et al., 1997; Wang et al., 1997; Xu et al., 1998). The cell nonautonomous effects of Hex might therefore be due, at least in part, to the induction of secreted Wnt antagonists, including, perhaps, Cerberus (reviewed by Niehrs, 1999). The simultaneous induction of a secreted molecule such as Cerberus and the cell-autonomous suppression of dorsal mesoderm fated to become prechordal plate could explain the anterior defects we observe in the phenotype depicted in Fig. $4 \mathrm{~F}$.

\section{Goosecoid as a direct target of Hex}

Several observations support a direct role for Hex in the regulation of Gsc. First, ectopic expression of Hex inhibits Gsc whereas Hex- $\lambda$ VP2 result in cell-autonomous induction of Gsc. Second, mutations in Hex- $\lambda$ VP2 that do not activate the Gsc promoter in ES cell culture do not induce Gsc in vivo. Third, both Hex and Hex- $\lambda$ VP2 can regulate Gsc in animal caps. Finally, during gastrulation stages, Gsc and Hex RNA are expressed in adjacent, but distinct, domains.

The finding that there is an antagonistic relationship between Hex and Gsc, two factors required for anterior patterning, suggests that head induction requires two distinct cell types. Cells expressing organiser markers such as Gsc and Chd would be distinct from anterior endodermal cells expressing $\mathrm{Hex}$ and Cerberus. In the absence of anterior endoderm expressing $\mathrm{Hex}$ and Cerberus, tissue expressing organiser markers may only form trunk derivatives. In support of this idea are recent findings showing that when chicken axial mesoderm expressing Chd and Gsc is cultured in vitro it forms notochord, but when recombined with anterior endoderm it forms prechordal plate mesoderm (C. Vesque, S. Ellis, P. Thomas, R. S. P. B. and M. Placzek, unpublished observations).

Inspection of published data on the mouse suggests that $\mathrm{Hex}$ and $G s c$ are initially co-expressed and that their domains, in both in visceral and definitive endoderm, then segregate (Belo et al., 1997; Blum et al., 1992; Shawlot and Behringer 1995; Wakamiya et al., 1997; Thomas et al., 1998; Wakamiya et al., 1998). Interestingly, marker analysis in mice with a targeted mutation in the Cripto locus suggests that the visceral endoderm domains of Gsc and Hex are distinct, even during the period in which the two genes are co-expressed. Thus, in null Cripto mutants, Hex-expressing cells remain at the distal tip of the egg cylinder (Ding et al., 1998). Consequently,
Cerberus is expressed at the distal tip whereas Gsc is only expressed at the embryonic-extraembryonic junction. In Xenopus, Chd and Gsc are expressed in a more superficial domain than Hex and Cerberus (Zorn et al., 1999).

\section{Conclusions}

Our experiments suggest that Hex regulates the formation of anterior structures by acting through both cell autonomous and cell non-autonomous routes. First, it suppresses expression of Gsc and Chd in a cell-autonomous (and, at least in the case of $G s c$, a direct) fashion, and in doing so it prevents the formation of organiser derivatives such as axial mesoderm. Secondly, and in an indirect manner, it activates the production of secreted factors which promote the formation of anterior structures. Such factors may include Cerberus or other Wnt inhibitors. In the absence of these factors, progeny of the classical Spemann organiser can form only trunk derivatives.

This work was supported by the Medical Research Council. We thank Mike Carey and Masa Tada for plasmids; Wendy Hatton and Andrew Stewart for technical assistance; Aldo Ciau-Uitz and Roger Patient for advice on sectioned in situs; Elena Casey, Tristan Rodriguez and Alex Gould for critical discussions of this manuscript; and Marysia Placzek, Courinne Hourart and Steve Wilson for communication of results prior to publication. J. M. B. was supported by a Human Frontiers Long Term Fellowship and the Medical Research Council.

\section{REFERENCES}

Astromoff, A. and Ptashne, M. (1995). A variant of lambda repressor with an altered pattern of cooperative binding to DNA sites. Proc. Natl. Acad. Sci. USA 92, 8110-8114.

Beddington, R. S. P., Morgernstern, J., Land, H. and Hogan, A. (1989). An in situ transgenic enzyme marker for the midgestation mouse embryo and the visualization of inner cell mass clones during early organogenesis. Development 106, 37-46.

Beddington, R. S. P. and Robertson, E. J. (1998). Anterior patterning in mouse. Trends Genet. 14, 277-284.

Beddington, R. S. P. and Robertson, E. J. (1999). Axis development and early asymmetry in mammals. Cell 96, 195-209.

Beddington, R. S. P. and Smith, J. C. (1993). Control of vertebrate gastrulation: inducing signals and responding genes. Curr. Opin. Genet. Dev. 3, 655-661.

Belo, J. A., Bouwmeester, T., Leyns, L., Kertesz, N., Gallo, M., Follettie, M. and De Robertis, E. M. (1997). Cerberus-like is a secreted factor with neutralizing activity expressed in the anterior primitive endoderm of the mouse gastrula. Mech. Dev. 68, 45-57.

Biben, C., Stanley, E., Fabri, L., Kotecha, S., Rhinn, M., Drinkwater, C., Lah, M., Wang, C. C., Nash, A., Hilton, D. et al. (1998). Murine cerberus homologue mCer-1: a candidate anterior patterning molecule. Dev. Biol. 194, 135-151.

Blum, M., Gaunt, S. J., Cho, K. W., Steinbeisser, H., Blumberg, B., Bittner, D. and De Robertis, E. M. (1992). Gastrulation in the mouse: the role of the homeobox gene goosecoid. Cell 69, 1097-1106.

Bouwmeester, T., Kim, S., Sasai, Y., Lu, B. and De Robertis, E. M. (1996). Cerberus is a head-inducing secreted factor expressed in the anterior endoderm of Spemann's organiser. Nature 382, 595-601.

Brickman, J. M., Adam, M. and Ptashne, M. (1999). Interactions between an HMG-1 protein and members of the rel family. Proc. Natl. Acad. Sci. USA 96, 10679-10683.

Carey, M., Lin, Y. S., Green, M. R. and Ptashne, M. (1990). A mechanism for synergistic activation of a mammalian gene by GAL4 derivatives. Nature 345, 361-364.

Cho, K. W., Blumberg, B., Steinbeisser, H. and De Robertis, E. M. (1991). Molecular nature of Spemann's organiser: the role of the Xenopus homeobox gene goosecoid. Cell 67, 1111-1120.

Chomczynski, P. and Sacchi, N. (1987). Single-step method of RNA isolation 
by acid guanidinium thiocyanate- phenol-chloroform extraction. Analyt. Biochem. 162, 156-159.

Cooke, J., Smith, J. C., Smith, E. J. and Yaqoob, M. (1987). The organization of mesodermal pattern in Xenopus laevis: experiments using a Xenopus mesoderm-inducing factor. Development 101, 893-908.

Crompton, M. R., Bartlett, T. J., MacGregor, A. D., Manfioletti, G., Buratti, E., Giancotti, V. and Goodwin, G. H. (1992). Identification of a novel vertebrate homeobox gene expressed in haematopoietic cells. Nucl. Acids Res. 20, 5661-5667.

Deardorff, M. A., Tan, C., Conrad, L. J. and Klein, P. S. (1998). Frizzled8 is expressed in the Spemann organiser and plays a role in early morphogenesis. Development 125, 2687-2700.

Ding, J., Yang, L., Yan, Y. T., Chen, A., Desai, N., Wynshaw-Boris, A. and Shen, M. M. (1998). Cripto is required for correct orientation of the anterior-posterior axis in the mouse embryo. Nature 395, 702-707.

Emami, K. H. and Carey, M. (1992). A synergistic increase in potency of a multimerized VP16 transcriptional activation domain. EMBO J. 11, 50055012 .

Fisher, J. P., Hope, S. A. and Hooper, M. L. (1989). Factors influencing the differentiation of embryonal carcinoma and embryo-derived stem cells. Exp. Cell Res. 182, 403-414.

Glinka, A., Wu, W., Delius, H., Monaghan, A. P., Blumenstock, C. and Niehrs, C. (1998). Dickkopf-1 is a member of a new family of secreted proteins and functions in head induction. Nature 391, 357-362.

Glinka, A., Wu, W., Onichtchouk, D., Blumenstock, C. and Niehrs, C. (1997). Head induction by simultaneous repression of Bmp and Wnt signalling in Xenopus. Nature 389, 517-519.

Harland, R. M. (1991). In situ hybridization: an improved whole-mount method for Xenopus embryos. Methods Cell Biol. 36, 685-695.

Hemmati-Brivanlou, A. and Thomsen, G. H. (1995). Ventral mesodermal patterning in Xenopus embryos: expression patterns and activities of BMP2 and BMP-4. Dev. Genet. 17, 78-89.

Henry, G. L. and Melton, D. A. (1998). Mixer, a homeobox gene required for endoderm development. Science 281, 91-96.

Ho, C. Y., Houart, C., Wilson, S. W. and Stainier, D. Y. (1999). A role for the extraembryonic yolk syncytial layer in patterning the zebrafish embryo suggested by properties of the hex gene. Curr. Biol. 9, 1131-1134.

Hoppler, S., Brown, J. D. and Moon, R. T. (1996). Expression of a dominantnegative Wnt blocks induction of $\mathrm{MyoD}$ in Xenopus embryos. Genes Dev. 10, 2805-2817.

Hudson, C., Clements, D., Friday, R. V., Stott, D. and Woodland, H. R. (1997). Xsox17alpha and -beta mediate endoderm formation in Xenopus. Cell 91, 397-405.

Ishikawa, T., Yoshioka, H., Ohuchi, H., Noji, S. and Nohno, T. (1995). Truncated type II receptor for BMP-4 induces secondary axial structures in Xenopus embryos. Biochem. Biophys. Res. Commun. 216, 26-33.

Itoh, K. and Sokol, S. Y. (1999). Axis determination by inhibition of Wnt signaling in Xenopus. Genes Dev. 13, 2328-2336.

Jones, C. M. Thomas, P., Q., Smith, J. C. and Beddington, R. S. P. (1999). An anterior signalling centre in Xenopus revealed by expression of the homeobox gene XHex. Curr. Biol. 9, 946-954.

Jones, C. M., Dale, L., Hogan, B. L., Wright, C. V. and Smith, J. C. (1996). Bone morphogenetic protein-4 (BMP-4) acts during gastrula stages to cause ventralization of Xenopus embryos. Development 122, 1545-1554.

Jones, C. M. and Smith, J. C. (1998). Establishment of a BMP-4 morphogen gradient by long-range inhibition. Dev Biol 194, 12-17.

Knoetgen, H., Viebahn, C. and Kessel, M. (1999). Head induction in the chick by primitive endoderm of mammalian, but not avian origin. Development 126, 815-825.

Leyns, L., Bouwmeester, T., Kim, S. H., Piccolo, S. and De Robertis, E. M. (1997). Frzb-1 is a secreted antagonist of Wnt signaling expressed in the Spemann organiser. Cell 88, 747-756.

Mailhos, C., Andre, S., Mollereau, B., Goriely, A., Hemmati-Brivanlou, A. and Desplan, C. (1998). Drosophila Goosecoid requires a conserved heptapeptide for repression of paired-class homeoprotein activators. Development 125, 937-947.

McMahon, J. A., Takada, S., Zimmerman, L. B., Fan, C. M., Harland, R. M. and McMahon, A. P. (1998). Noggin-mediated antagonism of BMP signaling is required for growth and patterning of the neural tube and somite. Genes Dev. 12, 1438-1452.

Mountford, P., Zevnik, B., Duwel, A., Nichols, J., Li, M., Dani, C., Robertson, M., Chambers, I. and Smith, A. (1994). Dicistronic targeting constructs: reporters and modifiers of mammalian gene expression. Proc. Natl. Acad. Sci. USA 91, 4303-4307.
Neidle, S. and Goodwin, G. H. (1994). A homology-based molecular model of the proline-rich homeodomain protein Prh, from haematopoietic cells. FEBS Lett. 345, 93-98.

Newman, C. S., Chia, F. and Krieg, P. A. (1997). The XHex homeobox gene is expressed during development of the vascular endothelium: overexpression leads to an increase in vascular endothelial cell number. Mech. Dev. 66, 83-93.

Niehrs, C. (1999). Head in the WNT: the molecular nature of Spemann's head organiser. Trends Genet. 15, 314-319.

Nieuwkoop, P. D. and Faber, J. (1994). Normal table of Xenopus laevis (Daudin): a systematical and chronological survey of the development from the fertilized egg till the end of metamorphosis. New York: Garland Publishing.

Ohashi, Y., Brickman, J. M., Furman, E., Middleton, B. and Carey, M. (1994). Modulating the potency of an activator in a yeast in vitro transcription system. Mol. Cell Biol. 14, 2731-2739.

Pearce, J. J., Penny, G. and Rossant, J. (1999). A mouse cerberus/Danrelated gene family. Dev. Biol. 209, 98-110.

Piccolo, S., Agius, E., Leyns, L., Bhattacharyya, S., Grunz, H., Bouwmeester, T. and De Robertis, E. M. (1999). The head inducer Cerberus is a multifunctional antagonist of Nodal, BMP and Wnt signals. Nature 397, 707-710.

Piccolo, S., Sasai, Y., Lu, B. and De Robertis, E. M. (1996). Dorsoventral patterning in Xenopus: inhibition of ventral signals by direct binding of chordin to BMP-4. Cell 86, 589-598.

Ptashne, M. (1992). A Genetic Switch: Phage [lambda] and Higher Organisms. Cambridge, Mass.: Cell Press: Blackwell Scientific Publications.

Rupp, R. A., Snider, L. and Weintraub, H. (1994). Xenopus embryos regulate the nuclear localization of XMyoD. Genes Dev. 8, 1311-1323.

Salic, A. N., Kroll, K. L., Evans, L. M. and Kirschner, M. W. (1997). Sizzled: a secreted XWnt8 antagonist expressed in the ventral marginal zone of Xenopus embryos. Development 124, 4739-4748.

Sasai, Y., Lu, B., Steinbeisser, H., Geissert, D., Gont, L. K. and De Robertis, E. M. (1994). Xenopus chordin: a novel dorsalizing factor activated by organiser- specific homeobox genes. Cell 79, 779-790.

Schier, A. F. and Gehring, W. J. (1992). Direct homeodomain-DNA interaction in the autoregulation of the fushi tarazu gene. Nature 356, 804807

Shawlot, W. and Behringer, R. R. (1995). Requirement for Lim1 in headorganiser function. Nature 374, 425-430.

Shawlot, W., Deng, J. M. and Behringer, R. R. (1998). Expression of the mouse cerberus-related gene, Cerr1, suggests a role in anterior neural induction and somitogenesis. Proc. Natl. Acad. Sci. USA 95, 6198-6203.

Slack, J. M. W., Dale, L. and Smith, J. C. (1984). Analysis of embryonic induction by using cell lineage markers. Phil. Trans. R. Soc. Lond. B 307, 331-336.

Smith, A. G., Heath, J. K., Donaldson, D. D., Wong, G. G., Moreau, J., Stahl, M. and Rogers, D. (1988). Inhibition of pluripotential embryonic stem cell differentiation by purified polypeptides. Nature 336, 688-690.

Smith, J. C. (1993). Purifying and assaying mesoderm-inducing factors from vertebrate embryos. In Cellular Interactions in Development: A Practical Approach (ed. D. A. Hartley), pp. xviii, 213. Oxford [England]; New York: IRL Press at Oxford University Press.

Smith, J. C. and Slack, J. M. W. (1983). Dorsalisation and neural induction: properties of the organiser in Xenopus laevis. J. Embryol. Exp. Morphol. 78, 299-317.

Smith, J. C. and Watt, F. M. (1985). Biochemical specificity of Xenopus notochord. Differentiation 29, 109-115.

Smith, S. T. and Jaynes, J. B. (1996). A conserved region of engrailed, shared among all en-, gsc-, Nk1-, Nk2- and msh-class homeoproteins, mediates active transcriptional repression in vivo. Development 122, 3141-3150.

Smith, W. C. and Harland, R. M. (1991). Injected XWnt-8 RNA acts early in Xenopus embryos to promote formation of a vegetal dorsalizing centre. Cell 67, 753-765.

Smith, W. C., Knecht, A. K., Wu, M. and Harland, R. M. (1993). Secreted noggin protein mimics the Spemann organiser in dorsalizing Xenopus mesoderm. Nature 361, 547-549.

Spemann, H. and Mangold, H. (1924). Über Induktion von Embrylonanlagen durch Implantation artfrmder Organisatoren. Wilhelm Roux Arch. Entw Mech. Org. 100, 599-683.

Symes, K. and Smith, J. C. (1987). Gastrulation movements provide an early marker of mesoderm induction in Xenopus laevis. Development 101, 339-349. Tada, M., O'Reilly, M. A. and Smith, J. C. (1997). Analysis of competence 
and of Brachyury autoinduction by use of hormone-inducible Xbra. Development 124, 2225-2234.

Tam, P. P. and Steiner, K. A. (1999). Anterior patterning by synergistic activity of the early gastrula organiser and the anterior germ layer tissues of the mouse embryo. Development 126, 5171-5179.

Tanaka, T., Inazu, T., Yamada, K., Myint, Z., Keng, V. W., Inoue, Y., Taniguchi, N. and Noguchi, T. (1999). cDNA cloning and expression of rat homeobox gene, Hex, and functional characterization of the protein. Biochem. J. 339, 111-117.

Thomas, P., Brickman, J. M., Popperl, H., Krumlauf, R. and Beddington, R. S. P. (1997). Axis duplication and anterior identity in the mouse embryo. Cold Spring Harb. Symp. Ouant. Biol. 62, 115-125.

Thomas, P. Q., Brown, A. and Beddington, R. S. P. (1998). Hex: a homeobox gene revealing peri-implantation asymmetry in the mouse embryo and an early transient marker of endothelial cell precursors. Development 125, 8594.

Treisman, J., Gonczy, P., Vashishtha, M., Harris, E. and Desplan, C. (1989). A single amino acid can determine the DNA binding specificity of homeodomain proteins. Cell 59, 553-562.

Wakamiya, M., Lindsay, E. A., Rivera-Perez, J. A., Baldini, A. and Behringer, R. R. (1998). Functional analysis of $\mathrm{Gscl}$ in the pathogenesis of the DiGeorge and velocardiofacial syndromes. Hum. Mol. Genet. 7, 1835 1840 .

Wakamiya, M., Rivera-Perez, J. A., Baldini, A. and Behringer, R. R.
(1997). Goosecoid and goosecoid-related genes in mouse embryogenesis. Cold Spring Harb. Symp. Quant. Biol. 62, 145-149.

Wang, S., Krinks, M., Lin, K., Luyten, F. P. and Moos, M., Jr. (1997). Frzb, a secreted protein expressed in the Spemann organiser, binds and inhibits Wnt-8. Cell 88, 757-766.

Watabe, T., Kim, S., Candia, A., Rothbacher, U., Hashimoto, C., Inoue, K. and Cho, K. W. (1995). Molecular mechanisms of Spemann's organiser formation: conserved growth factor synergy between Xenopus and mouse. Genes Dev. 9, 3038-3050.

Wilson, D., Sheng, G., Lecuit, T., Dostatni, N. and Desplan, C. (1993). Cooperative dimerization of paired class homeo domains on DNA. Genes Dev. 7, 2120-2134.

Xu, Q., D'Amore, P. A. and Sokol, S. Y. (1998). Functional and biochemical interactions of Wnts with FrzA, a secreted Wnt antagonist. Development 125, 4767-4776.

Yamanaka, Y., Mizuno, T., Sasai, Y., Kishi, M., Takeda, H., Kim, C. H., Hibi, M. and Hirano, T. (1998). A novel homeobox gene, dharma, can induce the organiser in a non-cell-autonomous manner. Genes Dev. 12, 2345-2353.

Yatskievych, T. A., Pascoe, S. and Antin, P. B. (1999). Expression of the homebox gene Hex during early stages of chick embryo development. Mech Dev. 80, 107-109.

Zorn, A. M., Butler, K. and Gurdon, J. B. (1999). Anterior endomesoderm specification in Xenopus by Wnt/beta-catenin and TGF-beta signalling pathways. Dev. Biol. 209, 282-297. 